\title{
High-accuracy adaptive modeling of the energy distribution of a meniscus-shaped cell culture in a Petri dish
}

\author{
Ignacio Gomez-Revuelto , Luis E. Garcia-Castillo , David Pardo \\ Departamento de Teoría de la Señal y Comunicaciones, Universidad Politécnica de Madrid, Madrid, Spain \\ Departamento de Teoria de la Señal y Comunicaciones, Universidad Carlos III de Madrid, Spain \\ Department of Applied Mathematics, Statistics, and Operational Research, University of the Basque Country (UPV/EHU), Spain \\ Basque Center for Applied Mathematics (BCAM), Spain \\ Ikerbasque (Basque foundation for sciences), Bilbao, Spain
}

\begin{abstract}
A B S T R A C T
Cylindrical Petri dishes embedded in a rectangular waveguide and exposed to a polarized electromagnetic wave are often used to grow cell cultures. To guarantee the success of these cultures, it is necessary to enforce that the specific absorption rate distribution is sufficiently high and uniform over the Petri dish. Accurate numerical simulations are needed to design such systems. These simulations constitute a challenge due to the strong discontinuity of electromagnetic material properties involved, the relative low field value within the dish cultures compared with the rest of the domain, and the presence of the meniscus shape developed at the liquid boundary. The latter greatly increases the level of complexity of the model in terms of geometry and intensity of the gradients/singularities of the field solution. In here, we employ a three-dimensional (3D) $h p$-adaptive finite element method using isoparametric elements to obtain highly accurate simulations. We analyze the impact of the geometrical modeling of the meniscus shape cell culture in the hp-adaptivity. Numerical results showing the error convergence history indicate the numerical difficulties arisen due to the presence of a meniscus-shaped object. At the same time, the resulting energy distribution shows that to consider such meniscus shape is essential to guarantee the success of the cell culture from the biological point of view.
\end{abstract}

\section{Introduction}

A Petri dish is a cylindrical plate often used for cell cultures. To induce and control the growth of these cell cultures, they may be exposed to various electromagnetic (EM) fields. One common scenario is to place the Petri dish into a rectangular waveguide that is illuminated with a polarized wave radiating at a particular frequency, $[1,18,17]$.

In order to ensure the proper growth of the cell cultures, it is necessary to guarantee the high and uniform distribution of the EM energy (typically measured in terms of SAR - Specific Absorption Rate-) within the Petri dish $[1,20]$. Some authors state that in addition to control the SAR, one also needs to impose some additional conditions, e.g., polarization, on the distribution of the full electromagnetic fields to secure the proper evolution of the cell culture (see [22] and references therein). Furthermore, the meniscus shape developed at the interface of the liquid with the dish provides a complex shape, whose geometry is typically expressed as a mathematical formula involving exponential and hyperbolic functions.

Suitable numerical simulation methods for these scenarios need to have the following features. First, they should be able to handle three-dimensional geometries, including the Petri dish shape, and the meniscus shape. Second, they should be able to efficiently deal with the discontinuous material properties at the air-liquid interface. Third, they should be flexible enough to enable simulation of all possible scenarios, including various geometries, polarizations, and frequencies. Finally, and more importantly, since the observed electromagnetic fields produced by modifications on the design system are often small but nonetheless important, the simulation software should be highly accurate for all considered models. Moreover, it should provide an error estimation in order to minimize uncertainty and guarantee the correctness of the solution for each model. 


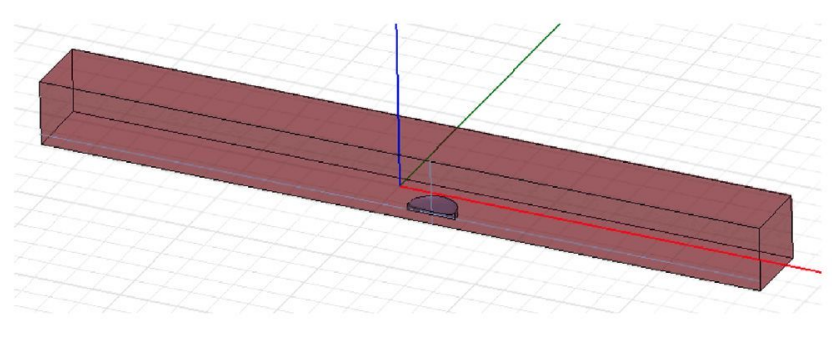

(a) Waveguide with Petri-dish.
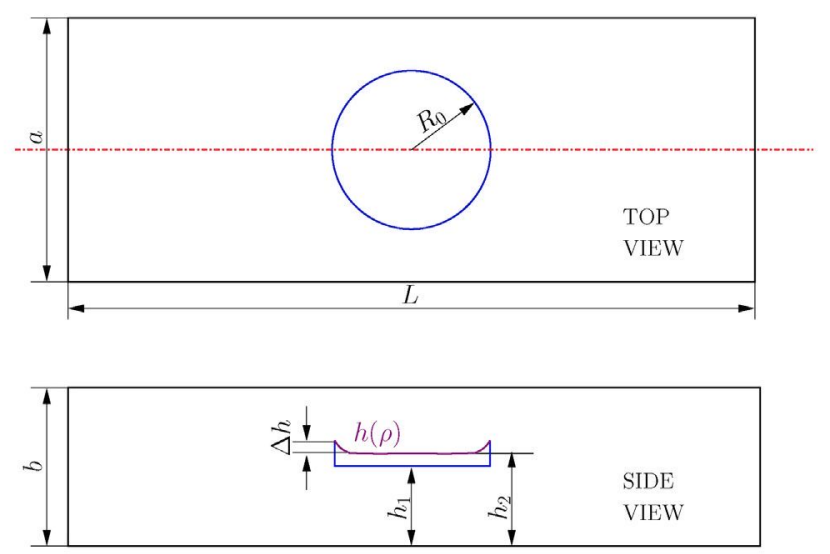

(b) Top and side views of the problem. Wide side $a$ of the waveguide ports correspond to $x$-axis. Side $b$ corresponds to $y$-axis. $z$-axis is along the waveguide.

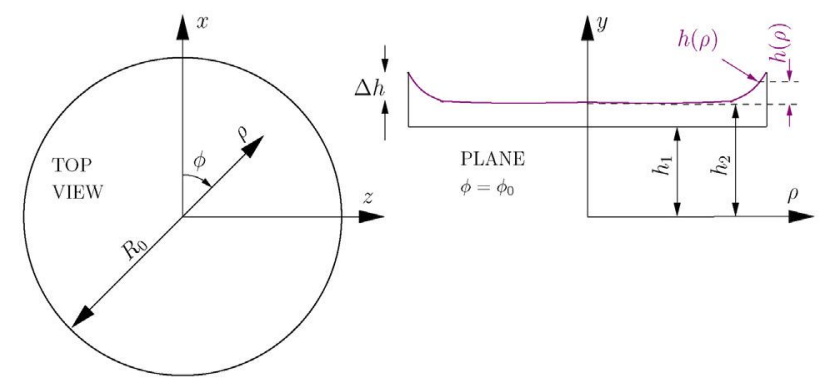

(c) Detail of meniscus profile: $h(\rho)=h_{2}+2 \Delta h e^{-\frac{R_{0}}{c}} \cosh \left(\frac{\rho}{c}\right)$

Fig. 1. Problem geometry. Dimensions expressed in millimeters: $a=95, b=45, R_{0}=16.9, L=300, h_{1}=27.5, h_{2}=30.5, \Delta h=2.51$.

Several numerical methods have been employed for Petri dish simulations exposed to EM fields in different configurations, e.g., $[3,20,21,16,1,22,23]$.Approaches based on differential formulations are mainly used because of their flexibility to deal with complex geometrical and material configurations. Among them, the most common numerical technique is finite differences (FD); typically, in time domain.

SAR data is obtained by averaging the energy distribution within a small cube shaped volume (known as voxel), which is the natural choice in FD grids. The presence of non-Cartesian geometries, boundary layers, and field singularities encountered on the resulting field solution together with internal resonances, requires the use of tiny voxels. Unfortunately, the use of small voxel sides of the order of one tenth (or even one hundredth) of a wavelength may not be enough in some cases to have confidence on the results. In those situations, the voxels close to the solid/liquid interface are skipped from the SAR distribution assessments, as it is reported in [22].
In here, we propose to employ a highly accurate method that works under all the above scenarios and provides an error estimation that guarantees the correctness of the solution. It is based on a Finite Element Method (FEM) that utilizes "adapted" meshes to both the geometry of the problem domain and its solution. A sequence of adapted meshes is generated in an automatic fashion by refining a given mesh in certain areas of the domain. Simultaneous $h$ and $p$ refinements, i.e., local variations of the element size $h$ and the polynomial order of approximation $p$ throughout the mesh are supported (the so called $h p$-adaptivity [5,6]). Preliminary results of the 3D implementation of the $h p$-adaptivity proposed by the authors [9] applied to the Petri dish problem were presented in [10], where the cell cultures were modeled as a circular dielectric, i.e., the meniscus shape was not included in the geometry. In this paper, the meniscus shape is included in the computational model. The main focus of this work is to analyze: (a) the numerical effect of the geometrical refinements in the $h p$-adaptivity due to the presence of a meniscus-shaped object, and (b) the differences obtained in the 


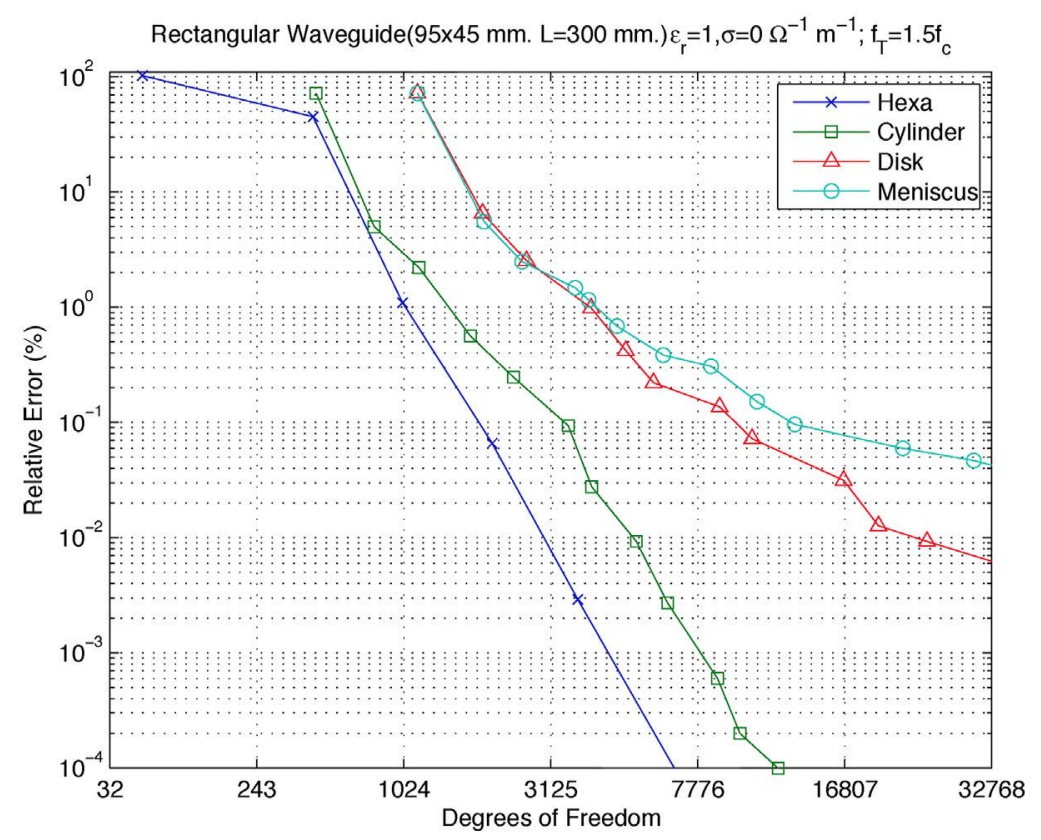

Fig. 2. Convergence history for the empty waveguide with different initial meshes.

physical results due to the presence of such complex geometrical object.

\section{Mathematical modeling and formulation}

We consider a Petri dish filled with a cell culture and placed inside a rectangular waveguide, as depicted in Fig. 1. Dimensions of the waveguide and dish are described Fig. 1. The meniscus shape is modeled as described in [20]. Its mathematical expression is displayed in Fig. 1 (we selected $c=2.01 \mathrm{~mm}$ ). The waveguide is electromagnetically excited with its fundamental mode, known as $\mathrm{TE}_{10}$ (details can be found in any of the numerous books in the subject, e.g., [4]). Operating frequency is $f=\frac{\omega}{2 \pi}=2.368 \mathrm{GHz}$.

The EM phenomena are governed by Maxwell's equations. By combining the two curl Maxwell's equations in the frequency domain the following double curl vector wave equation for the electric field $\mathbf{E}$ is obtained:

$\nabla \times \frac{1}{\mu_{r}} \nabla \times \mathbf{E}-k_{0}^{2} \varepsilon_{r} \mathbf{E}=0$

where $\varepsilon_{r}=\varepsilon / \varepsilon_{0}$ and $\mu_{r}=\mu / \mu_{0}$ being $\varepsilon, \mu$ the electric permittivity and magnetic permeability, respectively, of the medium, and $k_{0}=$ $\omega \sqrt{\varepsilon_{0} \mu_{0}}$. The subindex " 0 " is used to denote the vacuum medium. Symbol $\omega$ denotes angular frequency. Losses are modeled with a equal to $\sigma=2.3 \Omega^{-1} \mathrm{~m}^{-1}$ that in our problem corresponds to an imaginary part of the relative permittivity equal to 17.46 . The Petri dish itself is assumed to have the same electrical properties as that of the air; the dish has a relative permittivity much lower than that of the liquid contained within the dish, and its effect can be neglected, [14].

The excitation of the problem is imposed via a boundary condition at the incident port (boundary $\Gamma_{p}^{\text {inc }}$; left port in Fig. 1), namely:

$\hat{\mathbf{n}} \times \frac{1}{\mu_{r}} \nabla \times \mathbf{E}+j \frac{\beta_{10}}{\mu_{r}} \hat{\mathbf{n}} \times \hat{\mathbf{n}} \times \mathbf{E}=\mathbf{U}^{\text {in }}$, at the port boundaries

where $\beta_{10}$ is the propagation constant for the $\mathrm{TE}_{10}$ mode (equals to $\sqrt{5} \pi /(2 a)$ for the considered frequency) and $\mathbf{U}^{\mathrm{in}}=2 j\left(\beta_{10} / \mu_{r}\right) \hat{\mathrm{n}} \times$ $\hat{\mathbf{n}} \times \mathbf{E}^{\mathrm{in}}$ with $\mathbf{E}^{\mathrm{in}}$ the incident electric field of mode $\mathrm{TE}_{10}$ at the incident port. Symbol hi stands for the outward unit vector normal to the port. Boundary condition at the output port is given by the homogeneous version of Eq. (2).

The metallic walls of the waveguide (boundary $\Gamma_{D}$ ) are modeled as perfect electric conductors (PEC) by imposing the following Dirichlet boundary condition: $\hat{n} \times \mathbf{E}=0$.

The variational formulation is obtained by multiplying Eq. (1) with a test function $\mathbf{F}$. After integration by parts, it is obtained:

Find $\mathbf{E} \in \mathbf{W}$ such that

$$
\begin{aligned}
& \int_{V}(\nabla \times \mathbf{F}) \cdot\left(\frac{1}{\mu_{r}} \nabla \times \mathbf{E}\right) d \Omega-k_{0}^{2} \int_{V} \mathbf{F} \cdot \varepsilon_{r} \mathbf{E} d \Omega+\frac{j \beta_{10}}{\mu_{r}} \int_{\sum_{\Gamma_{p}^{i}}}(\hat{\mathrm{n}} \times \mathbf{F}) \cdot(\hat{\mathbf{n}} \times \mathbf{E}) d \boldsymbol{\Gamma} \\
& =2 \frac{j \beta_{10}}{\mu_{r}} \int_{\Gamma_{p}^{\mathrm{inc}}}(\hat{\mathrm{n}} \times \mathbf{F}) \cdot\left(\hat{\mathrm{n}} \times \mathbf{U}^{\mathrm{inc}}\right) d \Gamma \quad \forall \mathbf{F} \in \mathbf{W}, \quad \mathbf{W}:=\left\{\mathbf{A} \in \mathbf{H}(\mathbf{c u r l}, \Omega), \hat{\mathbf{n}} \times \mathbf{A}=0 \text { on } \Gamma_{D}\right\}
\end{aligned}
$$

\section{3. $h p$-FEM and automatic $h p$-adaptivity in 3D}

non-null electrical conductivity $\sigma$ that is introduced in the model through a complex permittivity, i.e., real parameter $\varepsilon$ turns into a complex parameter $\varepsilon_{C}=\varepsilon-j \sigma / \omega$.

In our problem, the air within the waveguide has no losses (i.e., $\sigma=0$ ), and the relative permeability and permittivity are equal to 1. The liquid contained within the Petri dish has a relative electric permittivity $\varepsilon_{r}=77$ and a relative magnetic permeability equal to 1. Losses in the liquid are modeled with an electrical conductivity
The 3D implementation of the hp-adaptivity proposed by the authors (see [9] and the references therein) is based on a selfadaptive strategy devised in [19] and further improved in [15,6]. The $h p$-adaptive strategy supports anisotropic refinements on irregular meshes with hanging nodes, and isoparametric elements as well as exact-geometry elements. It supports geometrical refinements through the use of its own geometrical package 


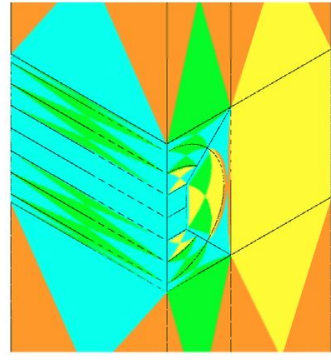

(a) Cylinder

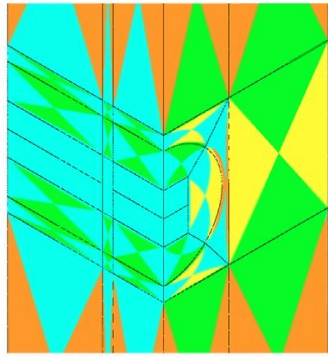

(b) Disc

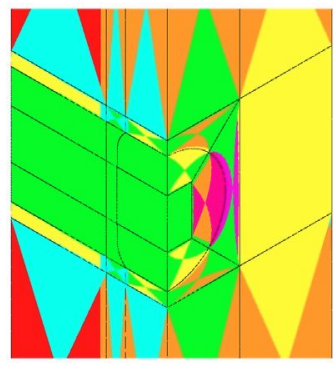

(c) Meniscus

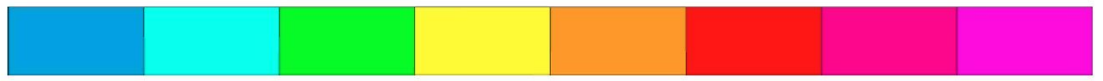

Fig. 3. Meshes corresponding to energy error $<0.1 \%$ (zoom).

to accurately model the problem geometry. Hexahedral $\mathbf{H}$ (curl) conforming finite elements satisfying the commutativity of the so-called de Rham diagram and hence, assuring convergence and stability of the numerical solution, are used, [8,7].

The main advantage of this method is that it provides exponential convergence rates in terms of the energy-norm error vs. the problem size when the right distribution of $h$ and $p$ throughout the computational domain is selected by the self-adaptive algorithm [2]. At the same time, it minimizes the dispersion error in wave propagation problems due to the use of high $p$ (see $[12,11,13]$ ).

\section{Numerical results}

We start by analyzing the effect of geometrical refinements that are needed to reproduce the geometry of the problem, namely, the Petri dish. Refinements forced to geometrically adapt to a complex geometry affect to the meshes delivered by the adaptivity and hence have impact on the numerical error of the finite element solution.

For that analysis, we consider the following four geometrical models: a Hexa (a waveguide without any object in its interior), a Cylinder (a waveguide containing a circular cylinder in the center), q Disc (a waveguide containing a thin disc in the center), Meniscus (a waveguide containing a meniscus-shaped object in the center). This latter geometry contains all the geometrical features needed for the proper modeling of the Petri dish and its liquid, while the remaining three geometrical models are successive approximations of the final model.

First, we consider the case of an empty waveguide, i.e., we select $\varepsilon_{r}=1$ and $\sigma=0$ everywhere in the domain (including the cell culture), so the exact solution corresponds to the $\mathrm{TE}_{10}$ field inside the waveguide, which is a smooth solution.

Fig. 2 shows the convergence history of the energy error corresponding to the four aforementioned initial geometries, which correspond to the same physical problem. The plot is displayed in a logarithmic scale in the ordinate axis, and in an algebraic scale

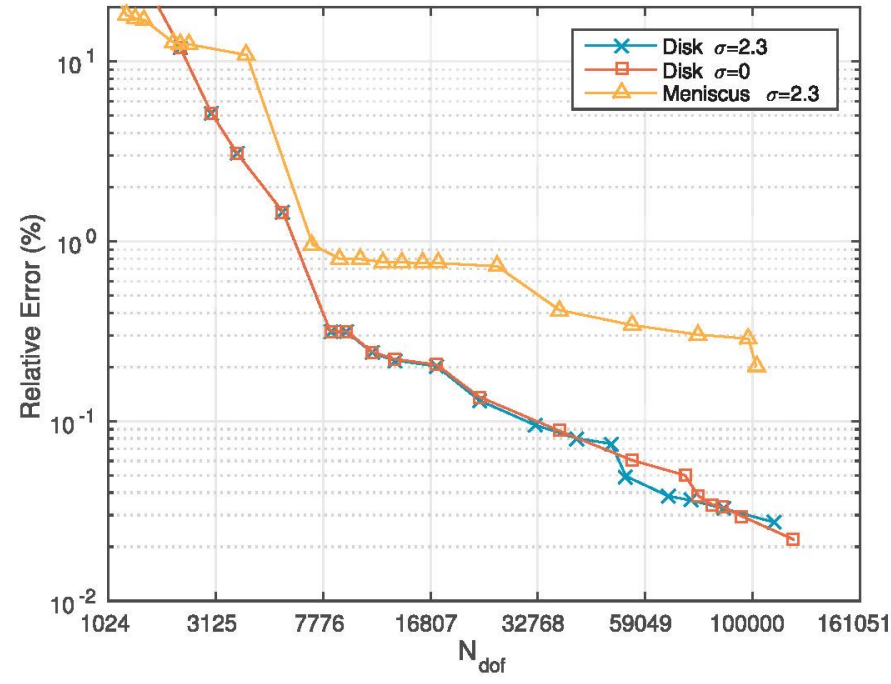

Fig. 4. Convergence history for the Petri dish (energy error).

in the abscissa axis. More precisely, the abscissa scale corresponds to $N_{\text {dof }}^{1 / 5}$ although the abscissa axis tics should be read as $N_{\text {dof }}$ in the plots; $N_{\text {dof }}$ is the number of unknowns. According to the theory developed in [2], the error associated to an optimal sequence of $h p$-grids behaves as $C \exp \left(-N_{\text {dof }}^{1 / 5}\right)$ in $3 \mathrm{D}$. Thus, a straight line in the considered scale indicates exponential convergence.

Fig. 2 confirms this exponential behavior in the cases of the "hexa" and "cylinder" initial meshes for the entire error range shown in the figure. Note that the adaptivity has been pushed beyond the limits of practical engineering error of $1 \%$. For the "disc" and "meniscus" initial meshes, the behavior is similar to the "hexa" and "cylinder" cases up to some error below 1\%. From that point on, a progressive deterioration in the slope is observed. 


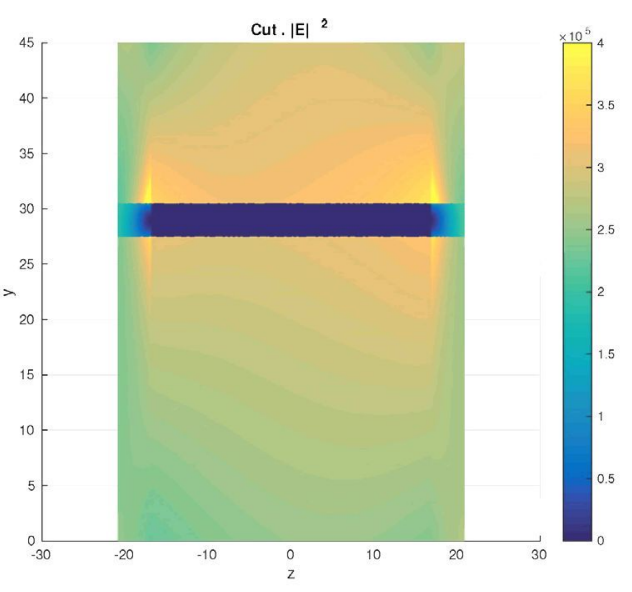

(a) Mesh after a few iterations

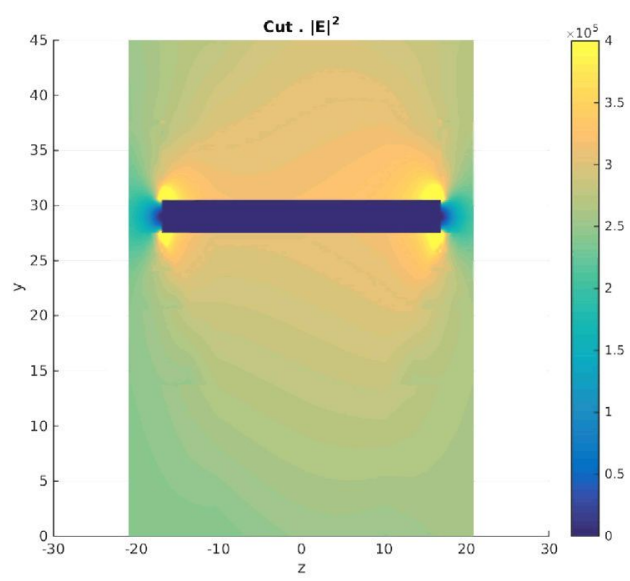

(c) Intermediate mesh

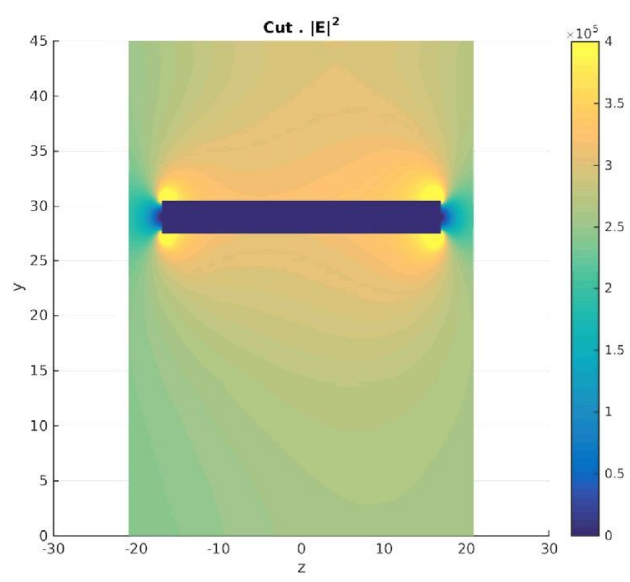

(e) Final mesh

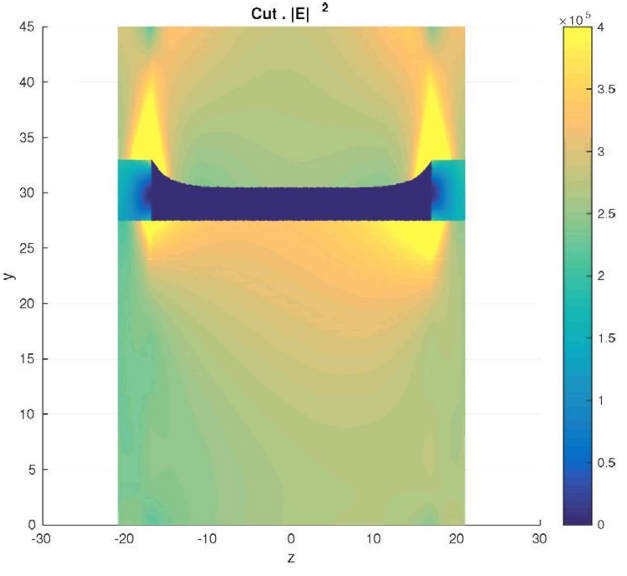

(b) Mesh after a few iterations

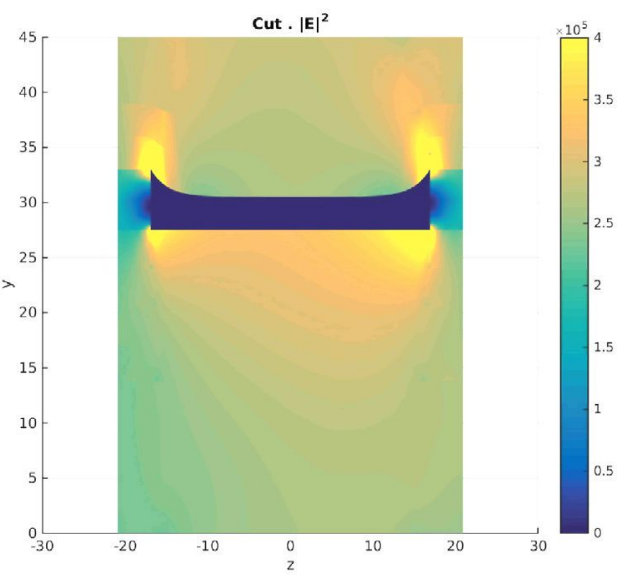

(d) Intermediate mesh

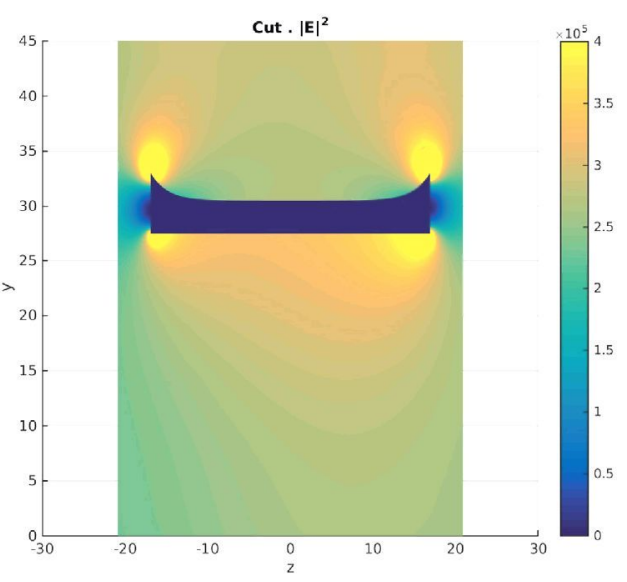

(f) Final mesh

Fig. 5. Energy distribution in the waveguide expressed as $|\mathrm{E}|^{2}\left(\mathrm{~V}^{2} / \mathrm{m}^{2}\right)$. Cut $y z$ of the waveguide. Meniscus effect included in the figures on the right.

Fig. 3 shows the final $h p$-meshes around the geometrically complex objects. The automatic $h p$-adaptive algorithm produces significantly higher orders of approximations for the meniscus-shaped case. The upper limit on the order of approximation (namely, $p=9$ ) existing in our implementation may explain some (but not all) of the observed convergence deterioration. The full reasons while such large convergence differences are observed when considering various geometrical objects with the same material properties are still unknown, and this is an active area of research.
We now consider the actual material properties corresponding to the Petri dish of the disc and meniscus-shaped objects, namely $\sigma=2.3 \mathrm{~S} / \mathrm{m}$ and $\epsilon_{r}=77$.

Fig. 4 shows the convergence history of the energy norm error. We conclude that material properties have a negligible effect on this problem in terms of convergence properties, while geometrical effects are notorious.

The importance of the geometry is also observed in the solution. Fig. 5 displays a plane cut on the yz plane of the energy distribution of the solution for various grids when considering both a disc 


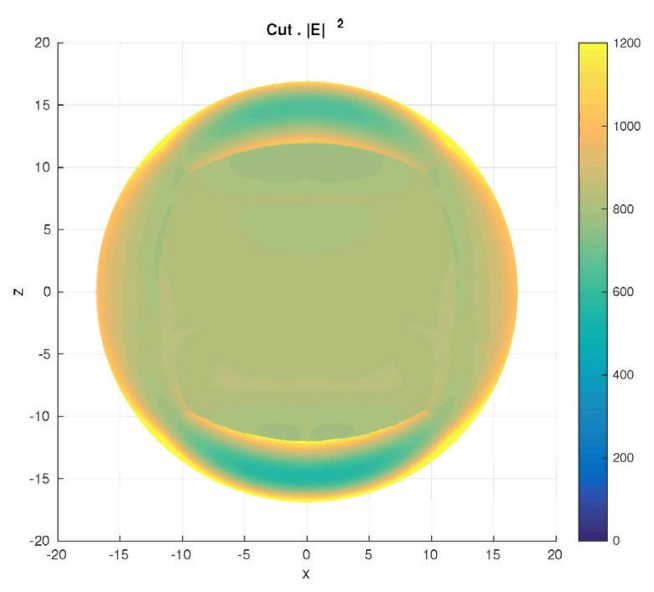

(a) Mesh after a few iterations

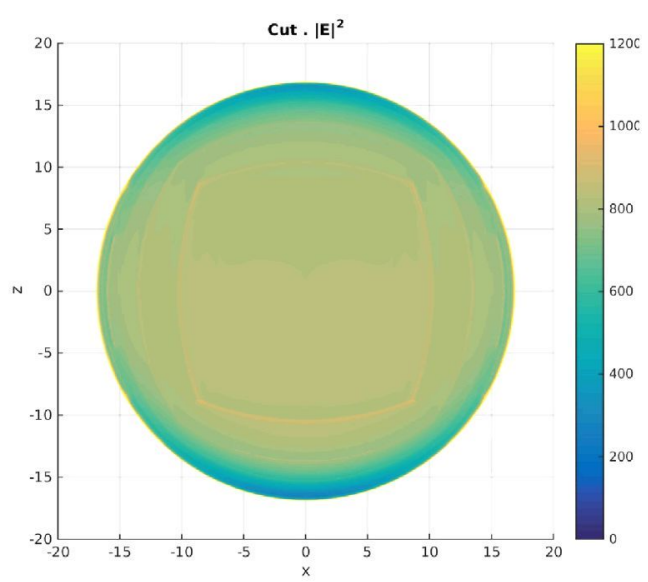

(c) Intermediate mesh

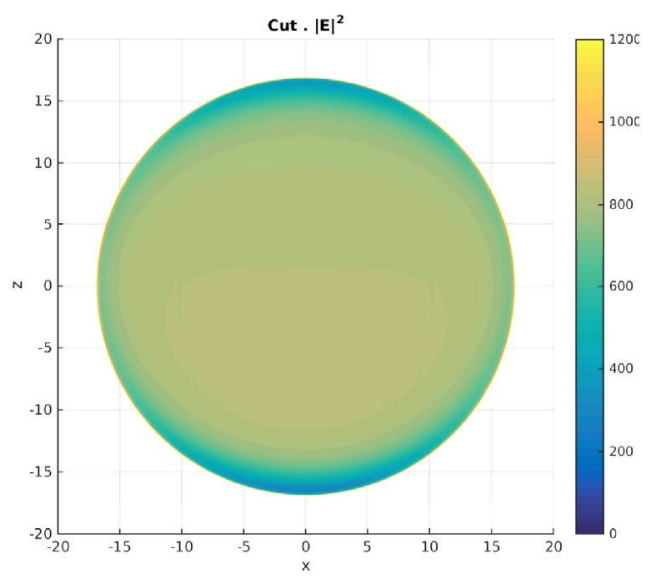

(e) Final mesh

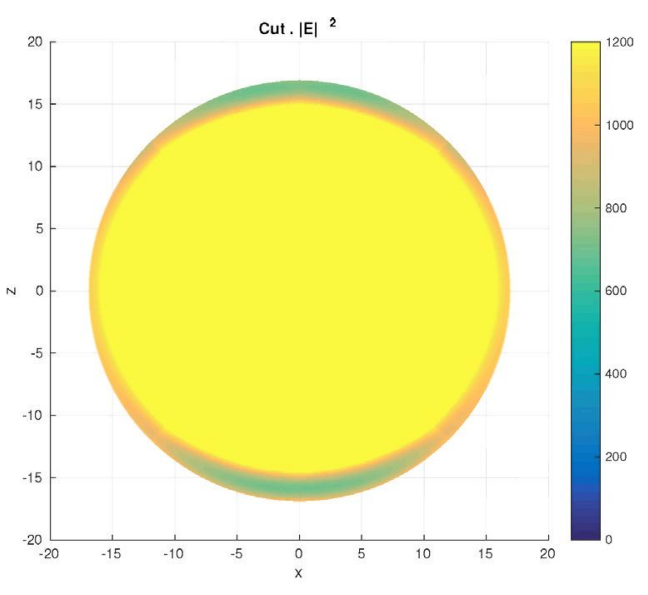

(b) Mesh after a few iterations

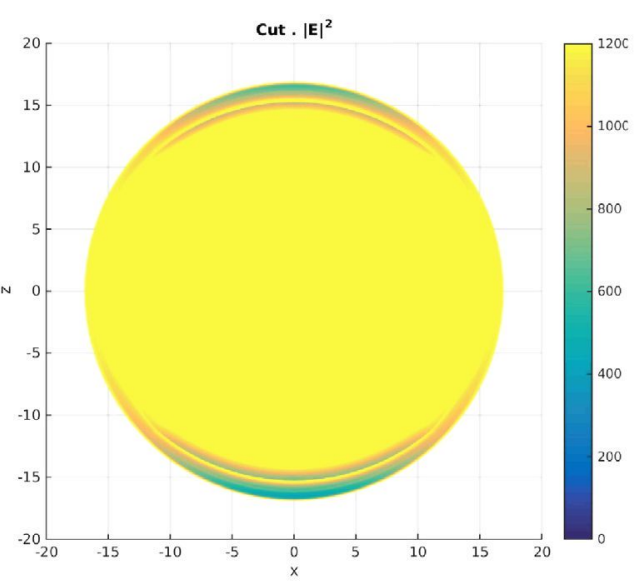

(d) Intermediate mesh

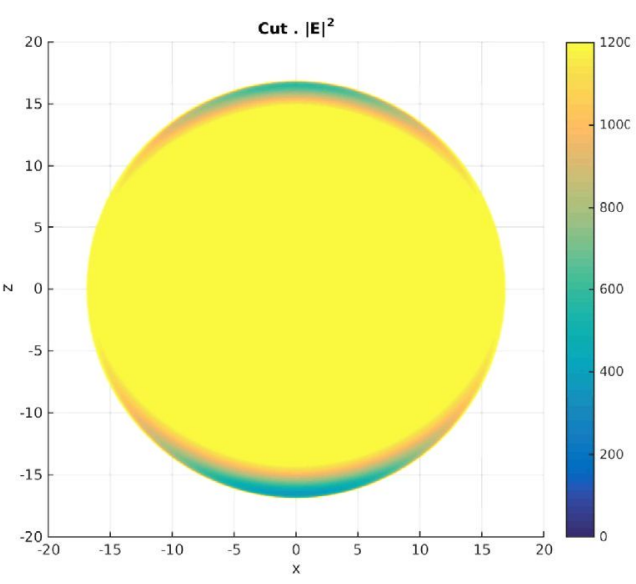

(f) Final mesh

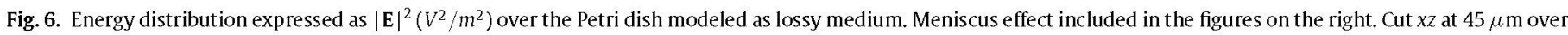
dish base.

and a meniscus-shaped Petri dish. For the meniscus-shaped object, a strong edge singularity develops along the top-exterior part of the Petri dish, as expected due to the reentrant corner contained on that geometry. The strength of this singularity is significantly lower in the disc-shaped object. Fig. 6 shows a plane cut on the $\mathrm{xz}$ plane of the energy distribution of the solution within the culture for the same meshes as those considered in Fig. 5. Again, significant differences are observed between the results corresponding to the disc and meniscus-shaped objects. For the later, we obtain a significantly larger (approx., 50\% more) energy distribution, as needed for biological purposes.

\section{Conclusions}

We have simulated the electromagnetic problem of the irradiation of a meniscus shaped cell culture within a Petri dish using an $h p$-adaptive FEM. The proposed method delivers highly accurate 
solutions with error control. The effect of the meniscus-shaped geometry that develops in the air/liquid interface significantly difficults its modeling, while it heavily influences the growth of the cell culture. The effect of the geometrical refinements in the performance of the adaptivity in such a complex geometrical object has been demonstrated.

\section{Acknowledgment}

The authors would like to thank Prof. González-García of University of Granada, Spain for providing the geometry of the waveguide with Petri dish. Also to Ph.D. student Adrián Amor for providing some numerical results used for code verification. The authors would like also to acknowledge the support of Ministerio de Educación y Ciencia of Spain under project TEC2010-18175/TCM. David Pardo has received funding from the European Union's Horizon 2020 research and innovation programme under the Marie Sklodowska-Curie grant agreement No. 644602, the Project of the Spanish Ministry of Economy and Competitiveness with reference MTM2013-40824-P, the BCAM "Severo Ochoa" accreditation of excellence SEV-2013-0323, the CYTED 2011 project 712RT0449, and the Basque Government through the BERC 2014-2017 program and the Consolidated Research Group Grant IT649-13 on "Mathematical Modeling, Simulation, and Industrial Applications (M2SI)".

\section{References}

[1] L.D. Angulo, S.G. Garcia, M.F. Pantoja, C.C. Sanchez, R.G. Martín, Improving the SAR distribution in Petri-dish cell cultures, J. Electromagn. Waves Appl. 24(5-6) (2010) 815-826.

[2] I. Babuška, B. Guo, Approximation properties of the $h p$-version of the finite element method, Comput. Methods Appl. Mech. Eng. 133 (1996) 319-346.

[3] M. Burkhardt, Numerical and experimental dosimetry of Petri dish exposure setups, Bioelectromagnetics 17 (1996) 483-493.

[4] R.E. Collin, Field Theory of Guided Waves, IEEE Press, 1991.

[5] L. Demkowicz, Computing with hp Finite Elements. I. One-and TwoDimensional Elliptic and Maxwell Problems, Chapman \& Hall/CRC Press, Taylor and Francis, 2007.

[6] L. Demkowicz, J. Kurtz, D. Pardo, M. Paszynski, W. Rachowicz, A. Zdunek, Computing with $h p$ Finite Elements. II Frontiers: Three Dimensional Elliptic and Maxwell Problems with Applications, Chapman \& Hall/CRC Press, Taylor and Francis, 2008.
[7] LF. Demkowicz, Finite element methods for Maxwell equations, in: Encyclopedia of Computational Mechanics, John Wiley \& Sons, Inc, 2004.

[8] L.F. Demkowicz, P. Monk, L. Vardapetyan, W. Rachowicz, De Rham diagram for hp finite element spaces, Comput. Math. Appl. 39(7-8) (2000) 29-38.

[9] I. Gomez-Revuelto, L.E. Garcia-Castillo, S. Llorente-Romano, D. Pardo, A threedimensional self-adaptive $h p$ finite element method for the characterization of waveguide discontinuities, Comput. Methods Appl. Mech. Eng. 249-252 (2012) $62-74$.

[10] I. Gomez-Revuelto, L.E. Garcia-Castillo, D. Pardo, High-accuracy adaptive simulations of a Petri dish exposed to electromagnet ic radiation, Proc. Comput. Sci. 18 (2013) 1555-1563.

[11] F. Ihlenburg, I. Babuška, Dispersion analysis and error estimation of Galerkin finite element methods for Helmhotz equation, Int. J. Numer. Methods Eng. 38 (1995) 3745-3774.

[12] F. Ihlenburg, I. Babuška, Finite element solution of the Helmholtz equation with high wave number. I: The $h$-version of the FEM, Comput. Math. Appl. 30 (9) (1995) 9-37.

[13] F. Ihlenburg, I. Babuška, Finite element solution of the Helmholtz equation with high wave number. II: the $h-p$-version of the FEM, SIAM J. Numer. Anal. $34(1)$ (1997) 315-358.

[14] T. Iyama, E. Hidetoshi, Y. Tarusawa, S. Uebayashi, M. Sekijima, T. Nojima, J. Miyakoshi, Large scale invitro experiment system for $2 \mathrm{GHz}$ exposure, Bioelectromagnetics 25 (2004) 599-606.

[15] J. Kurtz, L.F. Demkowicz, A fully automatic $h p$-adaptivity for elliptic PDEs in three dimensions, Comput. Methods Appl. Mech. Eng. 196 (2007) 3534-3545, http://dx.doi.org/10.1016/j.cma.2006.10.053

[16] M. Liberti, F. Apollonio, A. Paffi, M. Pellegrino, G. D'Inzeo, A coplanar-waveguide system for cells exposure during electrophysiological recordings, IEEE Trans. Microw. Theory Tech. 52 (11) (2004) 2521-2528.

[17] M.A. Martinez, M.A. Trillo, M.A. Cid, J.E. Page, A. Ubeda, Respuesta citostatica de celulas humanas NB69 a señales pulsadas en la banda de $2 \mathrm{GHz}$, in: In XXIII Simposium Nacional de la URSI, Madrid, España, 2008 September, pp. $70-74$.

[18] J.E. Page, El banco de irradiación in-vitro en la banda de $2 \mathrm{GHz}$ del hospital ramón y cajal, in: XXIII Simposium Nacional de la URSI, Madrid, España, 2008 September, pp. 51-55.

[19] W. Rachowicz, D. Pardo, L.F. Demkowicz, Fully automatic $h p$-adaptivity in three dimensions, Comput. Methods Appl. Mech. Eng. 195 (July (37-40)) (2006) $4186-4842$.

[20] J. Schuderer, N. Kuster, Effect of the meniscus at the solid/liquid interface on the SAR distribution in Petri dishes and flasks, Bioelectromagnetics 24 (2003) $103-108$.

[21] J. Schuderer, T. Samaras, W. Oesch, D. Spat, N. Kuster, High peak SAR exposure unit with tight exposure and environmental control for in vitro experiments at $1800 \mathrm{MHz}$, IEEE Trans. Microw. Theory Tech. 52 (8) (2004) 2057-2066.

[22] J.E. Varela, J.E. Page, J. Esteban, Design, implementation, and dosimetry analysis of an s-band waveguide in vitro system for the exposure of cell culture samples to pulsed fields, Bioelectromagnetics 31 (2010) 479-487.

[23] JX Zhao, H.M. Lu, J. Deng, Dosimetry and temperature evaluations of a $1800 \mathrm{MHz}$ TEM cell for in vitro exposure with standing waves, Prog. Electromagn. Res. (PIER) 124 (2012) 487-510. 